\title{
A NOTE ON A THEOREM OF MAY CONCERNING COMMUTATIVE GROUP ALGEBRAS
}

\author{
PAUL HILL AND WILLIAM ULLERY
}

(Communicated by Warren J. Wong)

\begin{abstract}
Let $G$ be a coproduct of $p$-primary abelian groups with each factor of cardinality not exceeding $\aleph_{1}$, and let $F$ be a perfect field of characteristic $p$. If $V(G)$ is the group of normalized units of the group algebra $F(G)$, it is shown that $G$ is a direct factor of $V(G)$ and that the complementary factor is simply presented. This generalizes a theorem of $\mathrm{W}$. May, who proved the result in the case when $G$ itself has cardinality not exceeding $\aleph_{1}$ and length not exceeding $\omega_{1}$.
\end{abstract}

Throughout let $F$ denote a perfect field of characteristic $p \neq 0$ and let $G$ denote a $p$-primary abelian group. In a recent paper, W. May [M2] proved the following result, where $V(G)$ denotes the group of normalized units in the group algebra $F(G)$; hence $V(G)=\left\{\sum c_{i} g_{i} \in F(G): \sum c_{i}=1\right\}$.

Theorem 1 ([M2]). If $|G| \leq \aleph_{1}$ and the length of $G$ does not exceed $\omega_{1}$, then $G$ is a direct factor of $V(G)$ and the complementary factor is simply presented.

Recall that a group is simply presented if it can be presented by generators and relations that involve at most two generators.

The main purpose of this note is to show that the condition on the length of $G$ in the above theorem can be omitted. In other words, the theorem is proved for a much more general class of groups (inasmuch as there exists an abundance of abelian $p$-groups of cardinality not exceeding $\aleph_{1}$ with arbitrary prescribed length $\left.\lambda<\omega_{2}\right)$. Actually, we extend the theorem to any abelian $p$-group $G$ which is a coproduct of groups with the cardinality of each factor not exceeding $\aleph_{1}$; of course such a $G$ can have arbitrarily large cardinality.

We refer to [M1] and [M3] as examples of the connection between the direct factor theorem and the isomorphism problem: When does $F(G) \cong F\left(G^{\prime}\right)$ imply that $G \cong G^{\prime}$ ?

As is customary for group algebras, we use the multiplicative notation for $G$ even though $G$ is abelian. Consequently, for an ordinal $\sigma, G^{\sigma}$ is the translation of the more familiar $p^{\sigma} G$; since there is only one relevant prime,

Received by the editors November $28,1989$.

1980 Mathematics Subject Classification (1985 Revision). Primary 20C07; Secondary 20K10.

Supported in part by NSF grant DMS 8800862. 
there is no ambiguity in the abbreviated notation. Thus we write $|x|=\sigma$ if $x \in G^{\sigma} \backslash G^{\sigma+1}$ while $|x|=\infty$ means that $x \in G^{\sigma}$ for every $\sigma$. Unless otherwise specified, $|x|$ will be computed in the largest containing group. If it is not clear from the context, we write $|x|_{G}$ to denote the height of $x$ computed in $G$. If $H \subseteq G$ we define the height spectrum of $H$ in $G$ to be the set of ordinals $\left\{|h|_{G}: h \in H\right\}$. The subgroup $H$ of the abelian $p$-group $G$ is isotype if $H \cap G^{\sigma}=H^{\sigma}$, and $H$ is nice in $G$ if $(G / H)^{\sigma}=G^{\sigma} H / H$, for all $\sigma$. If $H$ is both isotype and nice in $G$, it is said to be balanced. It is a well-known and not difficult fact that $G$ is a balanced subgroup of $V(G)$. Also, we mention that $H$ is nice in $G$ if and only if each coset of $H$ in $G$ contains an element with maximal height.

Since simply presented groups are always balanced projective, all that needs to be proved to establish the preceding theorem is that $V(G) / G$ is simply presented. Under the stated hypothesis, May [M2] was able to show that $V(G) / G$ is simply presented by using the Axiom 3 characterization of simply presented groups [F]; that is, May demonstrated that $V(G) / G$ has "sufficiently many" nice subgroups. This approach also captures the spirit of the present note. We identify and focus on certain nice subgroups of $V(G)$. In some cases, however, we state the preliminary results in the more general setting of arbitrary abelian $p$-groups. An example of this is our first lemma. Before stating this lemma, we need one more definition. Two subgroups $A$ and $B$ of the abelian $p$-group $G$ are called compatible and we write $A \| B$ if for each pair $(a, b) \in A \times B$ there exists $c \in A \cap B$ such that $|a c| \geq|a b|$. This notion was introduced in $[\mathrm{H}]$ and has played a significant role in a number of subsequent papers.

Lemma 1. Let $N$ be a subgroup of the p-group $G$. If each element of $G$ is contained in some subgroup $M$ with finite height spectrum in $G$ for which $N \| M$, then $N$ is nice in $G$.

Proof. For an arbitrary but fixed element $g \in G$, consider the set $S=\{|g x|$ : $x \in N\}$. By hypothesis, there exists a subgroup $M$ of $G$ containing $g$ for which $\{|x|: x \in M\}$ is finite and $N \| M$. Therefore, if $x \in N$, there exists $c \in N \cap M$ such that $|g c| \geq|g x|$. Since $g c \in M$, there are only a finite number of possibilities for $|g c|$ and each of these belongs to $S$. Consequently, $S$ must contain a largest element, and $N$ is nice in $G$.

If $\alpha=\sum c_{i} g_{i} \in F(G)$, with each $c_{i} \in F$ and $g_{i} \in G$, recall that the augmentation of $\alpha$, written $\operatorname{aug}(\alpha)$, is defined by $\operatorname{aug}(\alpha)=\sum c_{i}$. Thus, $V(G)=\{\alpha \in F(G): \operatorname{aug}(\alpha)=1\}$. In order to apply Lemma 1 and similar results to the group $V(G)$, we need to know that certain subgroups of $V(G)$ are compatible.

Lemma 2. Let $A$ and $B$ be arbitrary subgroups of $G$, then

(i) $G \| V(A)$,

(ii) $G V(A) \| V(B)$,

(iii) $G V(A) \| G V(B)$. 
Proof. Recall that if $\alpha=\sum c_{i} g_{i} \in V(G)$, where $c_{i} \neq 0$ and the $g_{i}$ 's are distinct elements of $G$, then $|\alpha|=\min \left\{\left|g_{i}\right|\right\}$. To verify part (i), simply take $\alpha=\sum c_{i} a_{i} \in V(A)$ with the $a_{i}$ 's distinct elements of $A$ and $c_{i} \neq 0$ in the field $F$ and consider $\alpha a_{1}^{-1}=c_{1}+\sum_{i>1} c_{i} a_{i} a_{1}^{-1}$. Observe that the height of $\alpha a_{1}^{-1}$ cannot be increased by multiplying by any group element $g \in G$, for $\left|\alpha a_{1}^{-1} g\right|>$ $\left|\alpha a_{1}^{-1}\right|$ implies on the one hand that $|g|=\left|\alpha a_{1}^{-1}\right|$, while on the other hand it implies that $|g|=\left|c_{1} g\right| \geq\left|\alpha a_{1}^{-1} g\right|>\left|\alpha a_{1}^{-1}\right|$. Clearly $a_{1}^{-1} \in G \cap V(A)$ and we have observed that $\left|\alpha a_{1}^{-1}\right| \geq\left|\alpha a_{1}^{-1}\left(a_{1} g\right)\right|=|\alpha g|$ for any $g \in G$. Therefore $G \| V(A)$. To prove that (ii) holds, let $\alpha=\sum c_{i} b_{i} \in V(B)$. By collecting the $b_{i}$ 's that are equal $\bmod A$, we can write $\alpha=E_{1} b_{1}+E_{2} b_{2}+\cdots+E_{n} b_{n}$, where $E_{i} \in F(A \cap B)$ and $b_{1}, b_{2}, \ldots, b_{n} \in B$ are distinct $\bmod A$. Since the augmentation of $\alpha$ is 1 , we may assume without loss of generality that $\operatorname{aug}\left(E_{1}\right)=c \neq 0$. Set $\beta=c^{-1} E_{1} b_{1}$ and observe that $\beta \in G V(A) \cap V(B)$. Moreover, $\alpha \beta^{-1}=c+E_{2}^{\prime} b_{2} b_{1}^{-1}+\cdots+E_{n}^{\prime} b_{n} b_{1}^{-1}$, where $E_{i}^{\prime}=E_{i}\left(c^{-1} E_{1}\right)^{-1} \in$ $F(A \cap B)$. From this equation it is easily seen that $\left|\alpha \beta^{-1}\right| \geq\left|\alpha \beta^{-1} \gamma\right|$ for any $\gamma \in G V(A)$. The key here, of course, is that $1, b_{2} b_{1}^{-1}, \ldots, b_{n} b_{1}^{-1}$ are distinct $\bmod A$. Hence $\left|\alpha \beta^{-1} \gamma\right|>\left|\alpha \beta^{-1}\right|$ leads to the contradiction

$$
\left|\alpha \beta^{-1} \gamma\right| \leq|c \gamma|=|\gamma|=\left|\alpha \beta^{-1}\right|<\left|\alpha \beta^{-1} \gamma\right| \text {. }
$$

This completes the proof of (ii), and an obvious modification of the preceding yields (iii).

Corollary 1 ([M2]). If $A$ is a subgroup of the p-group $G$, then $G V(A)$ is nice in $V(G)$.

Proof. Each element $\alpha$ of $V(G)$ is contained in $V(B)$ for some finite subgroup $B$ of $G$. Clearly $V(B)$ has finite height spectrum. By Lemma 2, $G V(A) \| V(B)$. Therefore, $G V(A)$ is nice as a consequence of Lemma 1.

The following is a generalization (perhaps only a slight one) of the preceding corollary.

Corollary 2. Let $N$ be a subgroup of $V(G)$ with $G \subseteq N \subseteq V(G)$. If $N \| G V(B)$ for every finite subgroup $B$ of $G$, then $N$ is nice in $V(G)$.

Proof. Under the hypothesis, we claim that $N \| V(B)$ for every finite subgroup $B$ of $G$. Choose $\alpha \in N$ and $\beta \in V(B)$, and let $|\alpha \beta|=\lambda$. Since $N \| G V(B)$, there exists $g \in G$ and $\beta^{\prime} \in V(B)$ such that $g \beta^{\prime} \in N$ and $\left|\alpha g \beta^{\prime}\right| \geq \lambda$. Note that $\beta^{\prime} \in N$ since $g \in G \subseteq N$ and that $\left|g \beta^{\prime} \beta^{-1}\right| \geq \lambda$. Using the fact that $G \| V(B)$ by virtue of Lemma 2, we can choose $b \in G \cap V(B)=B$ such that $\left|g b^{-1}\right| \geq \lambda$. From this and the inequality $\left|\alpha g \beta^{\prime}\right| \geq \lambda$, we conclude that $\left|\alpha b \beta^{\prime}\right| \geq \lambda$. Since $b \beta^{\prime} \in N \cap V(B)$, we have shown that $N \| V(B)$ for every finite subgroup $B$ of $G$. Lemma 1 implies that $N$ is nice in $V(G)$.

The next proposition is not required for the proofs of the main theorems, but it establishes a class of nice subgroups of $V(G)$ larger than the $G V(A)$ 's. 
Definition. A subgroup $S$ of $V(G)$ is called a solid subgroup if $\sum_{i \in I} c_{i} g_{i} \in$ $S$, with distinct $g_{i}$ 's in $G$, then $c^{-1} \sum_{j \in J} c_{j} g_{j} \in S$ whenever $J \subseteq I$ and $\operatorname{aug}\left(\sum_{j \in J} c_{j} g_{j}\right)=c \neq 0$.

It is not difficult to show that there exist solid subgroups of $V(G)$ containing $G$ which are not of the form $G V(A)$.

Proposition. If $G \subseteq S \subseteq V(G)$ and $S$ is a solid subgroup of $V(G)$, then $S$ is nice in $V(G)$.

Proof. With an argument very similar to that used in the proof of Lemma 2, we can show that $S \| G V(B)$ for every subgroup $B$ of $G$. Indeed, if $\alpha \in S$, we can write $\alpha=E_{1} g_{1}+E_{2} g_{2}+\cdots+E_{n} g_{n}$, where $E_{i} \in F(B)$ and the $g_{i}$ 's are distinct $\bmod B$. Without loss of generality, $\operatorname{aug}\left(E_{1}\right)=c \neq 0$. Observe that $c^{-1} E_{1} g_{1} \in G V(B) \cap S$ by virtue of $S$ being solid in $V(G)$. Let $\beta=c^{-1} E_{1} g_{1}$ and observe that $\alpha \beta^{-1}=c+E_{2}^{\prime} g_{2} g_{1}^{-1}+\cdots+E_{n}^{\prime} g_{n} g_{1}^{-1}$, where $E_{i}^{\prime} \in F(B)$. It should be clear now that $\left|\alpha \beta^{-1}\right| \geq\left|\alpha \beta^{-1} \gamma\right|$ whenever $\gamma \in G V(B)$, for the argument is essentially the same as in the case of Lemma 2 . Thus, for every $\delta \in G V(B),|\alpha \delta|=\left|\alpha \beta^{-1}(\beta \delta)\right| \leq\left|\alpha \beta^{-1}\right|$ and we have shown that $S \| G V(B)$. That $S$ is nice in $V(G)$ now follows from Corollary 2.

Only one more preliminary is needed for the proof of our first theorem, and we state it in the generality of arbitrary abelian $p$-groups.

Lemma 3. Suppose that $A$ and $B$ are compatible subgroups of the p-group $G$, where $A$ is nice in $G$ and $B$ has finite height spectrum in $G$. If $C$ is any subgroup of $B$, then $A C$ is nice in $G$.

Proof. Let $c \in C$. Since $A$ is nice in $G,|c A|_{G / A}=|c a|_{G}$ for some $a \in A$. But $A \| B$ implies that $|c d|_{G} \geq|c a|_{G}$ for some $d \in A \cap B$. Therefore, $|c A|_{G / A}=|c d|_{G}$ since $d \in A$ and heights cannot decrease under a homomorphic image. Since $c d \in B$ and $B$ has a finite height spectrum in $G$, we have demonstrated that $C A / A$ has a finite height spectrum in $G / A$. Therefore, $C A / A$ must be a nice subgroup of $G / A$. And since $A$ is nice in $G$, this implies that $A C$ is nice in $G$.

Recall that $F$ is a perfect field of characteristic $p \neq 0$ and $G$ is a $p$-primary abelian group.

Theorem 2. If $|G| \leq \aleph_{1}$, then $G$ is a direct factor of $V(G)$ and $V(G) / G$ is simply presented.

Proof. Let $\left\{A_{\alpha}: \alpha<\omega_{1}\right\}$ be a filtration of $G$; that is, we let

$$
1=A_{0} \subseteq A_{1} \subseteq \cdots \subseteq A_{\alpha} \subseteq A_{\alpha+1} \subseteq \cdots \quad\left(\alpha<\omega_{1}\right)
$$

be a smooth chain (smooth means $A_{\beta}=\bigcup_{\alpha<\beta} A_{\alpha}$ when $\beta$ is a limit) of countable subgroups of $G=\bigcup_{\alpha<\omega_{1}} A_{\alpha}$. For each $\alpha<\omega_{1}$, let $\left\{B_{\alpha, n}: n<\omega_{0}\right\}$ be a filtration of $A_{\alpha}$. In other words,

$$
1=B_{\alpha, 0} \subseteq B_{\alpha, 1} \subseteq \cdots \subseteq B_{\alpha, n} \subseteq \cdots \quad\left(n<\omega_{0}\right)
$$

is a chain of finite subgroups of $A_{\alpha}=\bigcup_{n<\omega_{0}} B_{\alpha, n}$. From Corollary 1, we know 
that the $G V\left(A_{\alpha}\right)$ 's form a chain of nice subgroups of $V(G)$. According to Lemma 3, $\left\{G V\left(A_{\alpha}\right) V\left(B_{\alpha+1, n}\right): n<\omega_{0}\right\}$ is a chain of nice subgroups of $V(G)$ going from $G V\left(A_{\alpha}\right)$ to $G V\left(A_{\alpha+1}\right)$. Moreover, Lemma 3 also implies that if $C$ is any subgroup between $V\left(B_{\alpha+1, n}\right)$ and $V\left(B_{\alpha+1, n+1}\right)$, then $G V\left(A_{\alpha}\right) C$ is nice in $V(G)$. Therefore, there is a smooth chain of nice subgroups of $V(G)$ between $G$ and $V(G)$, where the quotient of adjacent members of the chain is simple of order $p$. Since $G$ is balanced in $V(G)$, this means that $V(G) / G$ is simply presented and that $G$ is a direct factor of $V(G)$.

We separate part of the proof of our final theorem as the following lemma.

Lemma 4. If $H$ is a direct factor of $G$, then $G V(H) / G$ is a direct factor of $V(G) / G$. Consequently, $G V(H)$ is a balanced subgroup of $V(G)$.

Proof. The projection of $G$ onto $H$ induces a split epimorphism $V(G) \rightarrow$ $V(H)$. Thus, $V(G)=V(H) \times K$ for some subgroup $K$ of $V(G)$. From this it easily follows that $V(G) / G=G V(H) / G \times K G / G$. Finally, $G V(H)$ is balanced in $V(G)$, since $G$ is balanced in $V(G)$ and $G V(H) / G$ is balanced $V(G) / G$.

Theorem 3. Suppose $G=\bigsqcup_{i \in I} G_{i}$ is a coproduct of p-groups $G_{i}$. If $\left|G_{i}\right| \leq \aleph_{1}$ for all $i \in I$, then $G$ is a direct factor of $V(G)$ and $V(G) / G$ is simply presented. Proof. We show that $V(G) / G$ is simply presented by induction on $|G|$. If $|G| \leq \aleph_{1}$, the result follows from Theorem 2. Therefore, we may assume that $|G|>\aleph_{1}$. In this case, $|I|=|G|$. Let $\mu$ be the smallest ordinal of cardinality $|G|$; so we can take $I=\mu$ and $G=\bigsqcup_{i<\mu} G_{i}$. For each $\alpha<\mu$, set $H_{\alpha}=$ $\bigsqcup_{i<\alpha} G_{i}$.

Since $H_{\alpha}$ is a direct factor of $H_{\alpha+1}$, Lemma 4 implies that $H_{\alpha+1} V\left(H_{\alpha}\right) / H_{\alpha+1}$ is a direct factor of $V\left(H_{\alpha+1}\right) / H_{\alpha+1}$. By the induction hypothesis, the latter group is simply presented and hence so is

$$
V\left(H_{\alpha+1}\right) / H_{\alpha+1} V\left(H_{\alpha}\right) \cong\left(V\left(H_{\alpha+1}\right) / H_{\alpha+1}\right) /\left(H_{\alpha+1} V\left(H_{\alpha}\right) / H_{\alpha+1}\right) .
$$

Also by Lemma 4, $H_{\alpha+1} V\left(H_{\alpha}\right)$ is a balanced subgroup of $V\left(H_{\alpha+1}\right)$. Therefore, $V\left(H_{\alpha+1}\right)=H_{\alpha+1} V\left(H_{\alpha}\right) \times T_{\alpha}$ for some simply presented subgroup $T_{\alpha}$. Due to the smoothness of the sequence of $H_{\alpha}$ 's, it now follows easily that $V(G)=$ $G \times \amalg_{\alpha<\mu} T_{\alpha}$, thus proving the theorem.

\section{REFERENCES}

[F] L. Fuchs, Infinite abelian groups, vol. II, Academic Press, New York, 1973.

[H] P. Hill, Isotype subgroups of totally projective groups, Abelian Group Theory, Lecture Notes in Math., vol. 874, Springer-Verlag, Berlin, Heidelberg, and New York, 1981, pp. 305-321.

[M1] W. May, Unit groups and isomorphism theorems for commuiative group algebras, Groups and Semigroup Rings, North-Holland Math. Studies, no. 126, North-Holland, Amsterdam, 1986, pp. 163-178.

[M2] _ The direct factor problem for modular group algebras, Representation Theory, Group Rings, and Coding Theory, Contemp. Math., vol. 93, Amer. Math. Soc., Providence, RI, 1989, pp. 303-308.

[M3] _ Modular group algebras of simply presented abelian groups, Proc. Amer. Math. Soc. 104 (1988), 403-409. 